\title{
Protons in cubic yttria-stabilized zirconia: Binding sites and migration pathways
}

\author{
A.G. Marinopoulos \\ CFisUC, Department of Physics, University of Coimbra, Coimbra P-3004-516, Portugal
}

\section{A R T I C L E I N F O}

\section{Keywords:}

$\mathrm{Ab}$ initio

Oxides

Proton transport

Grain boundaries

\begin{abstract}
A B S T R A C T
Despite the fact that unusually high power generation attributed to proton conduction has been reported recently for nanostructured yttria-stabilized zirconia (YSZ) little is known about the atomistic mechanisms of proton transport and corresponding activation energies, namely the factors that dictate proton mobility. Important issues to be examined include the energetics of proton incorporation in the YSZ host, the binding of protons to intrinsic structural defects and the role of grain boundaries in proton conduction. The present study reports calculations based on density-functional theory (DFT) of defect-formation energies and energy barriers for proton migration in cubic YSZ for an yttria doping in the 10-12 mol\% range. For the bulk-crystalline lattice representative migration pathways are identified and the corresponding energy barriers for proton migration are calculated using the nudged elastic-band method. The feasibility for the grain boundaries to act as fast diffusion pathways for protons is also examined. For the high-angle $\Sigma 5(310)$ grain boundary DFT calculations were undertaken so as to determine the segregation propensity of protons as well as the magnitude of energy barriers for selected proton migration pathways at the core of this extended defect. The present calculations outline the importance of oxygen vacancies for proton migration in the bulk crystal and the ability of localized cation clusters at the grain-boundary core to act as strong obstacles to proton motion.
\end{abstract}

\section{Introduction}

Zirconia is an oxide with extensive applications in the ceramics technology [1]. It is routinely used as a thermal-barrier coating [2] and is an integral part in oxygen-sensor and abrasive components [3]. Zirconia is also commonly employed in its cubic phase as the solid electrolyte in fuel-cell technology and related applications in electrochemistry $[4,5]$. In these cases the cubic phase of zirconia is stabilized at lower temperatures by suitable aliovalent doping with metal oxides. The stabilization mechanism entails the formation of oxygen vacancies in the anion sublattice as charge-compensating defects [6]. Yttria-stabilized cubic zirconia (YSZ), in particular, is the zirconia-yttria solid solution most widely used in solid-oxide fuel cells owing to exceptional mechanical and chemical stabilities and high ionic conductivity.

Recent experimental studies initiated a strong interest in dense nanostructured YSZ as a proton conductor in solid-oxide fuel cells at low and intermediate temperatures [7-11]. Observations of the proton conductivity rapidly rising with decreasing grain size in nanostructured YSZ samples led to propositions that grain boundaries (GBs) are highly conductive paths for proton transport $[8,11]$. Other studies, however, contested such a point of view. Proton conduction was not observed through GBs in nanostructured YSZ [12]; instead it was found to mainly take place through the inner surface areas of the YSZ porous material [12]. Surface proton conduction was also documented to be much larger with respect to conduction in the bulk interior of YSZ or its internal GBs $[13,14]$.

These findings present a serious challenge for computational methodologies. Clearly, there is a need to have a consistent view of the proton-migration processes at the atomic level for both the bulk-crystalline phase and the GBs of YSZ. GBs are intrinsic extended defects within polycrystalline and nanocrystalline YSZ and it is vital to understand their role. The question is whether these defects are trapping regions for the protons, thus impeding their motion or, alternatively, whether they are fast-conduction pathways making proton migration easier.

The presence of protons dissolved in oxides has been well known since the early work by Stotz and Wagner [15]. Wagner [16] also studied the hydrogen solubility and diffusivity in zirconia-yttria solid solutions at $900{ }^{\circ} \mathrm{C}$ and $1000{ }^{\circ} \mathrm{C}$. The corresponding solubility was found to be proportional to the square root of the water partial pressure. The mole fraction of the protons in the oxide was determined to be about $2 \times 10^{-4}$ at $1000^{\circ} \mathrm{C}$ and $1 \mathrm{~atm}$ of $\mathrm{H}_{2} \mathrm{O}$ vapor. More recently, hydrogen configurations in YSZ have been studied by means of muonium spectroscopy $[17,18]$ and first-principles approaches [19] based on density-

E-mail address: marinop@teor.fis.uc.pt. 
functional theory (DFT) [20,21]. Hydrogen in its positively-charged state $\mathrm{H}^{+}$(namely the proton defect) adopted exclusively hydroxide $\mathrm{O}$ $\mathrm{H}$ configurations with the proton forming a short dative bond with the oxygen ions. From the calculated charge-transition levels it was deduced that hydrogen is a deep donor and an amphoteric impurity in YSZ with the pinning level, $E(+/-)$, positioned in the upper part of the band gap [19].

Proton transport in oxides is known to be accomplished through two distinct mechanisms [22,23]. First, via proton transfer whereby a proton initially bound to an oxygen ion hops to a neighboring oxygen ion forming a new O-H configuration. Secondly, via rotational diffusion of the protonic defect accomplished through a reorientation of the $\mathrm{O}-\mathrm{H}$ bond. This mechanism does not contribute directly to macroscopic diffusion since the proton remains bound to the same oxygen ion. Theoretical calculations of proton migration in crystalline phases of zirconia have been rare. For the monoclinic phase first-principles molecular dynamics calculations [24] showed that proton motion at lower temperatures is exclusively of the reorientation type. Proton hopping between neighboring oxygen ions was observed at higher temperatures ( $990 \mathrm{~K}$ ) and an activation energy of $0.32 \mathrm{eV}$ for individual proton jumps was deduced [24]. DFT-based metadynamics calculations [25] were also employed to study proton-diffusion events and trajectories in tetragonal zirconia at $1500 \mathrm{~K}$. For the cubic phase first-principles calculations of proton-migration barriers were only recently performed [26] and were restricted to the ideal fluorite structure, with an yttrium atom introduced substitutionally in the cation sublattice. The obtained energy barriers were mostly in the range from 0.32 to $0.42 \mathrm{eV}$ for selected proton diffusive jumps along certain prescribed pathways in the lattice.

Despite this initial progress at the atomistic level, important aspects of proton migration in YSZ are still unresolved: foremost of all the effect of oxygen vacancies to proton migration and secondly the magnitude of the reorientation step. Oxygen vacancies, in particular, are intrinsic structural defects of the equilibrium cubic YSZ phase and their effect on proton transport needs to be taken into account. The aim in the present study was to determine the relevant energetics of proton incorporation, the preferred binding sites of protons and the magnitude of their migration barriers in cubic YSZ from first principles. Similarly to earlier studies [19,27-32] YSZ was structurally modeled by special quasirandom structures simulating a stable zirconia-yttria solid solution with a $10.3 \mathrm{~mol} \% \mathrm{Y}_{2} \mathrm{O}_{3}$ doping. Representative migration paths for the proton were identified and the corresponding energy barriers were determined. The impact of the intrinsic structure of YSZ, in particular of the oxygen vacancies and local ionic environment and coordination was examined and discussed.

In order to assess the relevance of GBs in proton conduction further calculations were also performed. The aim in this case was to determine the segregation propensity of the protons at the core region of a highangle GB in this oxide and also to determine the magnitude of the energy barriers for proton migration. The $\Sigma 5(310) \mathrm{GB}$ was chosen for this purpose. This is a high-energy interface which has been frequently studied in the past experimentally by high-resolution electron microscopy and Z-contrast imaging [33] and theoretically by empirical [34,35] and first-principles [36-38] approaches. These studies resolved important structural patterns of the interfacial structure of this GB, in particular, the symmetry and projection of the cation sublattice along the [001] axis and the interface-plane termination.

\section{Theoretical background and preliminaries}

The calculations in the present study were based on spin-polarized DFT $[20,21]$ within the generalized-gradient approximation (GGA) for exchange and correlation, and the projector-augmented wave (PAW) method $[39,40]$. More specifically the semilocal PBE (proposed by Perdew, Burke and Ernzerhof) functional [41] was used. For this purpose, the corresponding implementations of the VASP code [42-44] were employed. The crystalline wavefunctions were expanded in a plane-wave basis limited by a cutoff energy of $470 \mathrm{eV}$. For both zirconium and yttrium atoms the semicore $4 s$ and $4 p$ electrons were included in the valence states. For the defect calculations, a Monkhorst-Pack [45] $2 \times 2 \times 2$ k-point mesh was chosen for the Brillouin-zone integrations.

The bulk-crystalline YSZ cubic phase was modeled by 96-atom quasi-random supercells with a lattice parameter twice as large as that of the primitive fluorite cell. The calculated magnitude for the latter $\left(\mathrm{a}_{\text {latt }}\right)$ was equal to $5.149 \AA$ after energy minimization with the PBE functional. An yttria doping of $10.3 \mathrm{~mol} \%$ was achieved by a suitable incorporation of yttria units in the ideal fluorite lattice. In the present case, six yttrium atoms were introduced substitutionally at random sites of the cation sublattice. These defects (denoted as $\mathrm{Y}_{\mathrm{Zr}}^{\prime}$ in Kröger-Vink notation) are negatively charged. Therefore, three oxygen vacancies were also created as charge-compensating defects of positive effective charge $\left(v_{0}^{*}\right)$. The incorporation reaction in Kröger-Vink notation is the following:

$\mathrm{Y}_{2} \mathrm{O}_{3} \stackrel{\mathrm{ZrO}_{2}}{\longrightarrow} 2 \mathrm{Y}_{\mathrm{Zr}}^{\prime}+\mathrm{v}_{\mathrm{O}}^{\bullet \bullet}+3 \mathrm{O}_{\mathrm{O}}^{\times}$

with $\mathrm{O}_{\mathrm{O}}^{\times}$denoting oxygen atoms at normal lattice sites.

Similarly to earlier atomistic calculations of YSZ [27-32] a number of different supercells were constructed starting with various vacancyvacancy distances and different vacancy-yttrium associations. Fifteen distinct supercells were finally obtained following energy minimization (see the Appendix). The lowest-energy supercell was then taken in order to perform the defect calculations. In this supercell the closestspaced oxygen vacancies were fifth-nearest neighbors.

For the GB calculations the high-angle $\Sigma 5(310)$ tilt GB was chosen. This is a high-energy interface with short in-plane periodicities and with the rotation (tilt) axis parallel to the [001] direction. A supercell of orthorhombic shape was constructed using periodic boundary conditions containing two equivalent GBs of alternating misorientations and totalling 228 atoms. The size of the supercell along the interface-normal vector was sufficiently large (equal to $2 \sqrt{10} \mathrm{a}_{\text {latt }}$ ) to minimize spurious interactions between the two GBs. Yttria units were introduced corresponding to $11.3 \mathrm{~mol} \%$ doping, thus approaching as close as possible the doping magnitude of the bulk-crystalline supercells. The incorporation of these units was done on a plane-by-plane basis ensuring a uniform distribution of yttrium atoms and oxygen vacancies in the whole supercell [38].

The formation energies, $\Delta E_{\text {def }}^{f}$, for the point defects studied, namely hydrogen and oxygen vacancy, were obtained as a function of the electron chemical potential $\mu_{\mathrm{e}}$ (Fermi energy) and of the chemical potentials $\mu_{\mathrm{i}}$ of the species involved. The corresponding expression is

$\Delta E_{\mathrm{def}}^{f}=E_{\mathrm{def}}^{\text {tot }}-E_{\mathrm{bulk}}^{\text {tot }}+(\Delta n) \mu_{\mathrm{i}}+q\left(\mu_{\mathrm{e}}+E_{\mathrm{V}}\right)$

where $E_{\mathrm{def}}^{\text {tot }}$ is the total energy of the defect supercell and $E_{\text {bulk }}^{\text {tot }}$ the total energy of the bulk supercell. $E_{\mathrm{V}}$ is the energy of the valence-band maximum and sets the reference for the positions of the Fermi level $\mu_{\mathrm{e}}$ in the gap. The magnitude of the latter, $E_{\text {gap }}$, was found equal to $3.91 \mathrm{eV} . \Delta n$ takes the value of -1 or +1 for the hydrogen or the vacancy defect, respectively. Finally, $q$ is the effective charge of the defect. A uniform compensating background charge was also added to the supercell when charged defects were studied. This allowed to describe such defects while enforcing the overall charge neutrality of the periodic supercells, both for bulk YSZ and the GB. The treatment of charged GBs would instead require a different approach and it was beyond the scope of the present study. Such GBs are always associated with adjoining space-charge layers [46] which provide charge compensation. The thickness of these layers can even reach up to several hundred nanometers; thus, it would require a large number of atoms to be properly described at the atomistic level.

The energy profiles for proton migration along several representative migration pathways in bulk YSZ and the GB were determined by means of the nudged elastic-band (NEB) method [47]. 


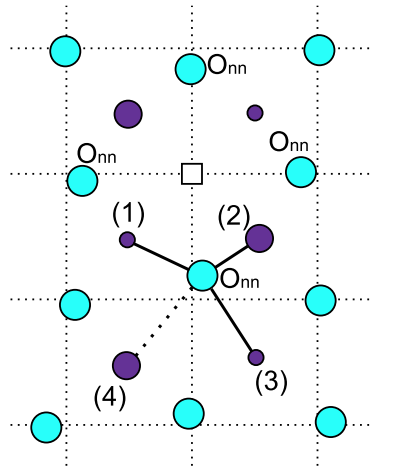

(a)

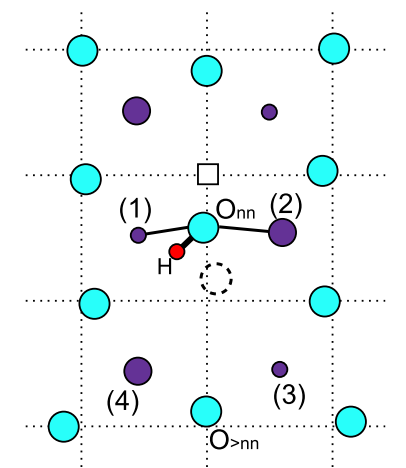

(b)

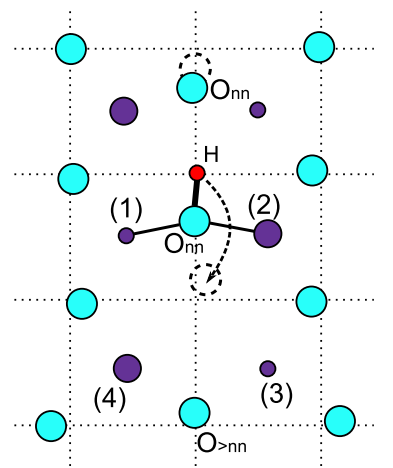

(c)

Fig. 1. (a) Part of the bulk-crystalline YSZ supercell. Distinct proton bond-type configurations near an oxygen vacancy (the latter denoted by the square symbol): (b) The proton $(\mathrm{H})$ resides near the vacant site and binds to a nearest-neighbor oxygen, $\mathrm{O}_{\mathrm{nn}}$. (c) The proton $(\mathrm{H})$ occupies the initially vacant site and binds to a nearest-neighbor oxygen, $\mathrm{O}_{\mathrm{nn}}$. The arrow denotes the vacancy jump to an adjacent cite of the anion sublattice. Oxygen ions are represented by the larger light-blue circles. Zirconium ions by the dark-violet circles. Protons $(\mathrm{H})$ are represented by the smaller red circles. The YSZ supercell is shown through a section of the cubic (100) plane near the region surrounding an oxygen-vacancy site. The projection is parallel to the [001] axis. All oxygen ions lie in the same cubic plane and have the same size. Zirconium ions of different size belong to successive cation planes parallel to the [001] axis. The $\mathrm{O}_{\mathrm{nn}}$ 's denote the oxygen nearest-neighbors to the vacancy. (1), (2), (3) and (4) denote the four nearest-

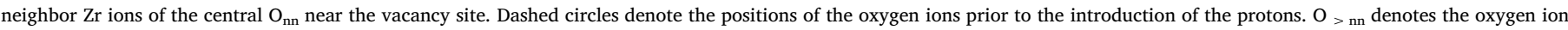

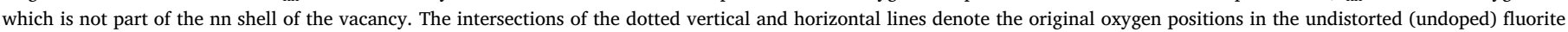
lattice. (For interpretation of the references to color in this figure legend, the reader is referred to the web version of this article.)
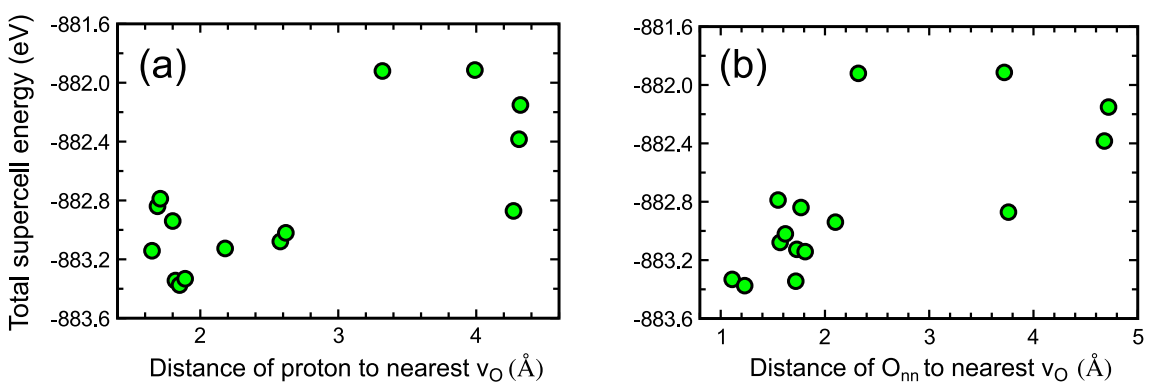

Fig. 2. Energies of all distinct proton configurations. (a) The energies are plotted with respect to the distances of the protons to their nearest oxygen vacancy $\mathrm{v}_{\mathrm{O}}$. (b) The energies are replotted with respect to the distances of $\mathrm{O}_{\mathrm{nn}}$ ions (the oxygen ions which form $\mathrm{O}-\mathrm{H}$ bonds with the protons) to their nearest oxygen vacancy $\mathrm{v}_{\mathrm{O}}$.

\section{Results}

\subsection{Defect-formation energies and proton sites in bulk YSZ}

The incorporation of the yttria units within the ideal (fluorite) cubic lattice entails certain changes to the local environment of the ions of both cation and anion sublattices. Whereas the $\mathrm{Zr}$ cations sustain rather small displacements away from their ideal fluorite sites (less than $0.25 \AA$ ), considerably larger distortions take place for the oxygen sublattice which can be as high as $0.8 \AA$ [19]. The most important relaxation mode involves the mutual attraction between the oxygen vacancies and their nearest-neighbor oxygen ions, $\mathrm{O}_{\mathrm{nn}}$. These ions are displaced predominantly towards the vacancy sites, along the [001] crystallographic directions (see Fig. 1 (a)). Such a relaxation mode has already been observed in previous first-principles calculations $[19,27,29,31,32]$ of cubic YSZ. Experimental observations by singlecrystal neutron diffraction also confirmed this specific relaxation pattern $[48,49]$.

A consequence of these strong displacements of the $\mathrm{O}_{\mathrm{nn}}$ ions is that some of them lose one of their Zr nearest neighbors; thus, they effectively possess a coordination number of three instead of the four-fold coordination of the ideal fluorite lattice. This can be seen in Fig. 1 (a) whereby the $\mathrm{O}_{\mathrm{nn}}$ is seen bonded to only three $\mathrm{Zr}$ nearest neighbors with corresponding $\mathrm{d}_{\mathrm{Zr}-\mathrm{O}}$ distances in the 2.10 to $2.30 \AA$ range (depicted by solid lines). The fourth lost $\mathrm{Zr}$ neighbor is found further away from $\mathrm{O}_{\mathrm{nn}}$ (at $2.89 \AA$; connected to it by a dotted line). By comparison, the corresponding $\mathrm{Zr}-\mathrm{O}$ nearest-neighbor distances in the undistorted fluorite lattice are equal to $2.24 \AA$. This relaxation pattern brings about a mixed (three-fold and four-fold) cation coordination for the oxygen ions in cubic YSZ, similarly to monoclinic zirconia [24] which is the thermodynamically stable phase of pure zirconia at $0 \mathrm{~K}$.

Following the structural optimization of the lowest-energy bulkcrystalline supercell, a single hydrogen atom $(\mathrm{H})$ was introduced interstitially at various positions in the lattice. In addition to neutral atomic hydrogen $\left(\mathrm{H}^{0}\right)$ the other two charge states of hydrogen $(q=+1$ and -1 ) were modeled by fixing the total electron occupation in the supercell after removing and adding one electron, respectively. Minimization of the total supercell energy was then performed to determine the equilibrium sites of hydrogen in the host as well as the respective formation energies for each of its charge states.

In agreement with previous studies [19,24,26,50-53] of proton incorporation in bulk-crystalline zirconia phases, protons $\left(\mathrm{H}^{+}\right)$were observed to associate with the oxygen ions as hydroxide ions $\left(\mathrm{OH}_{\mathrm{O}}{ }^{\circ}\right)$ by forming short dative $\mathrm{O}-\mathrm{H}$ bonds. In total, fifteen distinct proton configurations were obtained in the bulk supercell with $\mathrm{O}-\mathrm{H}$ bond lengths in the $0.98-1.00 \AA$ range. A second oxygen ion was also found reasonably close to the proton, within a much wider distance ranging from $1.70 \AA$ to $2.15 \AA$, depending upon the configuration. Examination of the energetics of these fifteen configurations showed that the final proton sites are not isoenergetic; the respective energies are plotted in Fig. 2. It can be seen that the corresponding range of energies is about $1.5 \mathrm{eV}$. It can also be seen that protons possessed consistently lower energies when residing closer to the oxygen vacancies, in particular to within a range of distances from 1.60 to $2.60 \AA$ from the nearest vacancy. From the total energies replotted in a different manner (see Fig. 2 (b)) it can also be inferred that protons bind more favorably to the oxygen ions, $\mathrm{O}_{\mathrm{nn}}$, which are nearest neighbors to the vacancies (see Fig. 1).

Very likely the smaller coordination numbers for the $\mathrm{O}_{\mathrm{nn}}$ 's near the vacancies is a decisive factor. In most cases, these $\mathrm{O}_{n n}-\mathrm{H}$ configurations possessed lower formation energies by as much as $0.5 \mathrm{eV}$ with respect to O-H configurations formed further away from the vacancies. It is also noteworthy that the mean yttrium-proton distance for the former cases is $3.36 \AA$, whereas the mean yttrium-proton distance is smaller ( $2.60 \AA)$ for the latter configurations. This is not surprising since the yttrium atoms occupied second nearest-neighbor positions with respect to the oxygen vacancies in the YSZ supercell (at least $4.2 \AA$ away).

Protons were also observed to occupy initially vacant oxygen sites while forming an $\mathrm{O}-\mathrm{H}$ bond with an $\mathrm{O}_{\mathrm{nn}}$ ion (see Fig. 1 (c)). In such 
cases the $\mathrm{O}_{\mathrm{nn}}$ ions displace so much that effectively create another vacant site in the anion sublattice. Such a process can be considered as a spontaneous migration of the initial oxygen vacancy to an adjacent site of the lattice.

A common structural characteristic of all proton configurations forming near the oxygen vacancies is a pronounced displacement of the oxygen ions $\mathrm{O}_{\mathrm{nn}}$ (that bond with the proton) towards the nearest oxygen vacancy. The magnitude of these displacements was recorded to be as much as $1 \AA$ in many cases and as a result a $\mathrm{Zr}$-O bond is broken in the process (see Fig. 1). In particular, for the configurations shown in Fig. 1 (b) and (c) the $\mathrm{O}_{\mathrm{nn}}$ ions displaced by $1.09 \AA$ and $1.18 \AA$, respectively, following the proton incorporation in the lattice. In certain cases, even $\mathrm{O}_{\mathrm{n}}$ 's which do not bind to the protons also displaced towards the vacancies (see Fig. 1 (c)). This structural relaxation pattern leads to a decrease of the coordination numbers of the $\mathrm{O}_{\mathrm{nn}}$ 's: they only have two $\mathrm{Zr}$ nearest neighbors in a bridge-type $-\mathrm{Zr}-\mathrm{O}_{\mathrm{nn}}-\mathrm{Zr}$ - bonding pattern (see Fig. 1).

Neutral $\left(\mathrm{H}^{0}\right)$ and negatively-charged hydrogen $\left(\mathrm{H}^{-}\right)$stabilize, in general, at different positions within the YSZ lattice [19]. $\mathrm{H}^{0}$ adopts two distinct configurations: in the lower-energy one hydrogen forms a short covalent bond with the oxygen ions, resembling structurally the configurations of the protonic $\left(\mathrm{H}^{+}\right)$defects. For these cases the excess electron is of $4 d$ character with the electron density localized predominantly near neighboring $\mathrm{Zr}$ ions in the vicinity of hydrogen. Instead, the higher-energy $\mathrm{H}^{0}$ configurations are atom-like with hydrogen occupying the interstitial cube-centered $(c c)$ sites or the vacancy $\left(\mathrm{v}_{\mathrm{O}}\right)$ sites with the $1 s$ electron centered on the hydrogen nucleus. Negativelycharged hydrogen $\left(\mathrm{H}^{-}\right)$was found to reside most favorably at the $c c$ and $\mathrm{v}_{\mathrm{O}}$ sites [19].

By taking the lowest-energy configuration for each charge state of hydrogen the respective formation-energy plot was obtained as a function of the Fermi-level $\left(\mu_{\mathrm{e}}\right)$ position in the gap (see Fig. 3 (a)). The formation energies were also obtained for the two extremes of the hydrogen chemical potential, $\mu_{\mathrm{H}}$, that correspond to hydrogen-rich and hydrogen-poor conditions. For the former conditions hydrogen is assumed to be in equilibrium with molecular $\mathrm{H}_{2}$ gas (g). The chemical potential of hydrogen in this case attains its highest value $\mu_{\mathrm{H}}^{\mathrm{o}}$, namely: $\mu_{\mathrm{H}} \leq \mu_{\mathrm{H}}^{\mathrm{o}}$. Using the present DFT-PBE functional it is calculated as half the total energy of an isolated hydrogen molecule at $0 \mathrm{~K}$ : $\mu_{\mathrm{H}}^{\mathrm{o}}=\frac{1}{2} \mathrm{E}^{\text {tot }}\left(\mathrm{H}_{2}\right)$. On the other hand, the magnitude of $\mu_{\mathrm{H}}$ is also constrained by the formation of water, $\mathrm{H}_{2} \mathrm{O}(\mathrm{g})$. In particular, in equilibrium with water and oxygen gas and under wet oxidizing conditions, $\mu_{\mathrm{H}}$ reaches its lower limit, obtained as

$\mu_{\mathrm{H}}=\frac{1}{2}\left(\mu_{\mathrm{H}_{2} \mathrm{O}}^{o}-\mu_{\mathrm{O}}^{\mathrm{o}}\right)$

where the chemical potential $\mu_{\mathrm{H}_{2} \mathrm{O}}^{o}$ is the DFT-PBE total energy of an isolated water molecule at $0 \mathrm{~K}$. The chemical potential for oxygen, $\mu_{\mathrm{O}}^{\mathrm{o}}$, on the other hand is taken as $\mu_{\mathrm{O}}^{0}=E_{\mathrm{O}}^{\text {tot }}-\frac{1}{2} E_{\mathrm{O}_{2}}^{\text {bind }}$, with $E_{\mathrm{O}_{2}}^{\text {bind }}$ being the experimental binding energy (equal to $5.22 \mathrm{eV}$ ) of the $\mathrm{O}_{2}$ molecule and $E_{O}^{\text {tot }}$ the total energy of an isolated spin-polarized oxygen atom within
DFT-PBE. This procedure avoids the well-known overbinding of the $\mathrm{O}_{2}$ molecule within GGA functionals [54].

The lower limit of $\mu_{\mathrm{H}}$ (hydrogen-poor conditions) is more realistic since in most experimental situations the YSZ samples were in contact with water vapor $[7-9,11,16]$.

Inspection of the formation-energy plot for hydrogen (see Fig. 3 (a)) shows that the neutral state, $\mathrm{H}^{0}$, is never thermodynamically stable for any $\mu_{\mathrm{e}}$ value in the gap. Instead, only the ionized charge states of hydrogen are favored. Proton $\left(\mathrm{H}^{+}\right)$, in particular, is the thermodynamically stable state from the valence-band edge $\left(E_{\mathrm{V}}\right)$ up to the pinning level, $E(+/-)$, where the most stable charge state of hydrogen becomes $\mathrm{H}^{-}$. Within the present DFT-PBE approach the position of the pinning level is $E(+/-)=E_{\mathrm{C}}-1.47 \mathrm{eV}$. It can also be seen that the magnitude of formation energies is higher for hydrogen-poor conditions. Therefore, it will be more difficult for hydrogen to incorporate in the YSZ lattice when the oxide sample is in contact with $\mathrm{H}_{2} \mathrm{O}$ vapor.

The formation energies can also be calculated at finite temperature $T$ and for different partial pressures $p$ of the surrounding gas through the dependence of the chemical potentials of the respective gas phases upon $T$ and $p$. Namely,

$\mu(T, p)=\mu^{o}+\Delta \mu\left(T, p^{o}\right)+k_{\mathrm{B}} T \ln \left(\frac{p}{p^{o}}\right)$

where $\mu^{o}$ is the value of the chemical potential obtained from the DFT$\mathrm{PBE}$ calculations at $0 \mathrm{~K}$. The temperature-dependent $\Delta \mu\left(T, p^{o}\right)$ term contains the gas enthalpy and entropy and is calculated from thermochemical data [55]. $p^{o}$ is the reference standard-state pressure (usually taken as $1 \mathrm{~atm}$ ) and $k_{\mathrm{B}}$ the Boltzmann constant. For hydrogen-poor conditions the corresponding defect-formation energies at two different temperatures, $500 \mathrm{~K}$ and $1200 \mathrm{~K}$, and assuming partial pressures $p_{\mathrm{H}_{2}} \mathrm{O}$ and $p_{\mathrm{O}_{2}}$ equal to $1 \mathrm{~atm}$ were determined. For these conditions the respective change $\Delta \mu$ in the chemical potential of hydrogen (entering Eq. (3)) was found equal to $-0.20 \mathrm{eV}$ and $-0.585 \mathrm{eV}$ for $500 \mathrm{~K}$ and $1200 \mathrm{~K}$, respectively. The final results are shown in Fig. 3 (a). The formation energy is seen to increase with increasing T; thus, it becomes less favorable to introduce hydrogen to the YSZ lattice from the environment.

To determine the equilibrium proton concentration $\left(c_{\mathrm{H}^{+}}\right)$in YSZ one would have to consider also the formation energies of all possible competing defects (and their charge states) which can form at the different sites of the host lattice [57-59]. In the present study a simpler approach is instead adopted; the proton concentration $c_{\mathrm{H}^{+}}$is obtained by means of a Boltzmann distribution involving solely the Gibbs formation energy $\left(\Delta \mathrm{G}_{\mathrm{def}}^{f}\right)$ of the protonic defect at specified $(T, p)$ conditions. Such a procedure, however, may not be a good approximation at high temperatures or extreme oxygen partial-pressure conditions [58].

Accordingly, $c_{\mathrm{H}^{+}}$is expressed as number of protonic defects per formula unit of zirconia: $c_{\mathrm{H}^{+}}=n_{\mathrm{D}} \times \exp \left(-\Delta G_{\mathrm{def}}^{f} / \mathrm{k}_{\mathrm{B}} \mathrm{T}\right)$, where $\mathrm{n}_{\mathrm{D}}$ represents the number of possible defect sites in the lattice per formula unit. It is assumed that protons bind to each oxygen ion by adopting (a) interstitial hydrogen

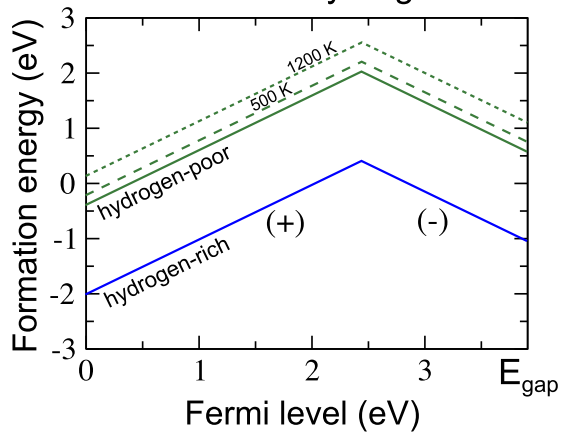

(b)

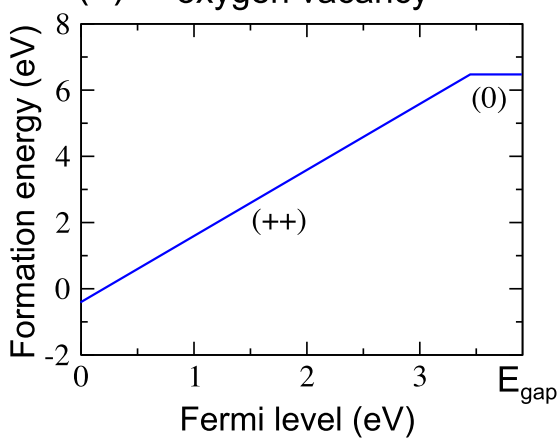

Fig. 3. (a) Formation energy of interstitial hydrogen for the two extremes of the hydrogen chemical potential, corresponding to hydrogen-rich and hydrogen-poor conditions. For the latter case the corresponding results for finite temperature were also plotted: for $500 \mathrm{~K}$ (dashed line) and for $1200 \mathrm{~K}$ (dotted line). (b) Formation energy of oxygen vacancy for oxygen-rich conditions (oxidation limit). The reference for the Fermi level $\left(\mu_{\mathrm{e}}\right)$ is the valence-band top, $\mathrm{E}_{\mathrm{V}}$. $\mathrm{E}_{\mathrm{gap}}$ denotes the edge of the conduction band, $\mathrm{E}_{\mathrm{C}}$. 
two configurations on the average. $\Delta G_{\mathrm{def}}^{f}$ can be approximated from the calculated defect-formation energies obtained from Eq. (1) adding also a contribution from the formation entropy $S_{\text {def }}^{f}$ of the proton. The entropy $S_{\text {def }}^{f}$ mainly originates from the change of the vibrational frequencies after the introduction of the defect in the lattice. The final expression is

$\Delta G_{\mathrm{def}}^{f}=\Delta E_{\mathrm{def}}^{f}-T S_{\mathrm{def}}^{f}$

A constant (temperature-independent) formation entropy of $5 k_{\mathrm{B}}$ was assigned to the protonic defect. This value is similar to reported entropies of point defects in solids [56] that were calculated within a harmonic approximation to the lattice dynamics. Assuming contact with water vapor (hydrogen-poor conditions) $\Delta G_{\mathrm{def}}^{f}$ can then be obtained as a function of the electron chemical potential, $\mu_{\mathrm{e}}$. The exact magnitude of $\mu_{\mathrm{e}}$, however, is not an independent variable. In practice, it depends upon doping and atmospheric conditions. From theoretical means its strict calculation requires the self-consistent solution of the charge-neutrality equation for each atmospheric condition [57-59]. This equation depends upon the concentrations and charge states of all competing point defects as well as the electron and hole concentrations in the crystal. Here, the value of $\mu_{\mathrm{e}}$ is assumed to lie at mid-gap, something that has been established experimentally for YSZ samples by electron-spectroscopy techniques [60]. For this magnitude of $\mu_{\mathrm{e}}$ the calculated $c_{\mathrm{H}^{+}}$equals $1.65 \times 10^{-15}$ and $6.96 \times 10^{-7}$ proton defects per zirconia formula unit at $500 \mathrm{~K}$ and $1200 \mathrm{~K}$, respectively.

The equilibrium of YSZ with water vapor can also be examined through the hydration reaction $[15,16,61,62]$ whereby protons may incorporate in the lattice via dissociative absorption of water from the environment, as follows:

$\mathrm{H}_{2} \mathrm{O}(\mathrm{g})+\mathrm{v}_{\mathrm{O}}^{\bullet \bullet}+\mathrm{O}_{\mathrm{O}}^{\times} \rightleftharpoons 2 \mathrm{OH}_{\mathrm{O}}^{\bullet}$

Namely, an oxygen vacancy is filled by the oxygen atom of the water molecule and two protonic defects are created in the YSZ lattice. In order to calculate the free energy of hydration, $\Delta G_{\text {hydr }}$, the formation energy of the oxygen vacancy in YSZ was determined. Introducing oxygen vacancies as nearest neighbors to the existing vacancies in the supercell led to highenergy, unstable configurations involving strong structural relaxation effects with the vacancies migrating to neighboring sites. Similar spontaneous vacancy jumps have also been observed in previous DFT calculations of vacancy-vacancy interactions in YSZ $[32,63]$ and should be expected for solids such as YSZ which are oxygen-ion conductors. Instead, the creation of an oxygen vacancy avoiding close proximity to the existing ones led to favorable configurations of lower formation energies. The corresponding results for one of these latter cases are plotted in Fig. 3 (b) for oxygen-rich conditions (oxidation limit), namely: $\mu_{\mathrm{O}}=\mu_{\mathrm{O}}^{\mathrm{o}}$.

From Fig. 3 (b), it can be seen that the oxygen vacancy is positively charged ( +2 charge state) in large parts of the gap, with the $E(++/ 0)$ transition level found near the conduction band, at $\mathrm{E}_{\mathrm{C}}-0.47 \mathrm{eV}$. In contrast, the singly-positive charge state is never thermodynamically stable for any values of $\mu_{\mathrm{e}}$. These results are qualitatively similar to previous DFT calculations for the oxygen vacancy in the cubic and tetragonal phases of zirconia $[27,53,64]$. The formation energy of the vacancy in its neutral state is equal to $6.46 \mathrm{eV}$ at Fermi-level positions close to the conduction band (n-type conditions), whereas it decreases when approaching the valence band attaining its lowest-value $(-0.43 \mathrm{eV})$ for $\mu_{\mathrm{e}}=0$ ( $p$-type conditions).

In the present study, the free energy of hydration, $\Delta G_{\mathrm{hydr}}$, was approximated by the hydration enthalpy at $0 \mathrm{~K}, \Delta E_{\text {hydr }}^{\text {tot }}$. The basic assumption here is that the vibrational-energy contribution in the solid largely cancels the gas-phase contribution to the $\Delta G_{\text {hydr }}$. Detailed first-principles calculations of the free energy of hydration in $\mathrm{BaZrO}_{3}$ showed the validity of such approximation at temperatures near $0 \mathrm{~K}$ [65].

Accordingly, $\Delta E_{\text {hydr }}^{\text {tot }}$ was determined from the balance of the defectformation energies in the hydration reaction $[59,65]$ as follows:
$\Delta E_{\mathrm{hydr}}^{\text {tot }}=2 \Delta E_{\mathrm{OH}_{\mathrm{O}}^{*}}^{f}-\Delta E_{\mathrm{v}_{\mathrm{O}}}^{f}$

The final result for $\Delta E_{\text {hydr }}^{\text {tot }}$ was equal to $-0.13 \mathrm{eV}$. The negative sign means that hydration is an exothermic process in YSZ at $0 \mathrm{~K}$ and protons can be favorably incorporated in the lattice from the surrounding water vapor. It is also important to note that the obtained value of $\Delta E_{\text {hydr }}^{\text {tot }}$ should be considered approximate owing to the fact that both $\Delta E_{\mathrm{OH}_{\mathrm{O}}^{*}}^{f}$ and $\Delta E_{\mathrm{v}_{\mathrm{O}}}^{f}$ exhibit a range of magnitudes depending upon the position of the respective defects within the supercell. For $\Delta E_{\mathrm{OH}_{0}^{*}}^{f}$, in particular, the lowest-energy configuration was chosen (see Fig. 2), thus rendering the calculated $\Delta E_{\text {hydr }}^{\text {tot }}$ more negative.

The small magnitude of $\Delta E_{\text {hydr }}^{\text {tot }}$ means that further water uptake of YSZ is not expected to occur at finite temperatures. With increasing temperature the entropy decrease associated with the absorption of water molecules from the atmosphere dominates the hydration reaction $[61,65]$ and will make the free energy of hydration positive, namely $\Delta G_{\text {hydr }} \geq 0$. Consequently, there will be no driving force for water to be accommodated in the lattice from the surrounding gas. This result is in accordance with previous experimental observations for stabilized-zirconia ionic conductors which were found to dissolve very little amounts of water in their structure $[16,66]$. Recent DFT calculations [63] have reported small and positive hydration energies for YSZ suggesting that hydration will not occur at all.

\subsection{Proton migration pathways in bulk YSZ}

Having determined an adequate number of minimum-energy proton configurations the next step was to identify representative pathways for proton migration that connect adjacent proton positions. Two distinct categories of proton-migration paths were identified. First, near-vacancy paths which remain localized near an oxygen-vacancy site. The pathways labelled $\mathrm{P}_{1}$ and $\mathrm{P}_{2}$ in Fig. 4 comprise such path types. Pathway $\mathrm{P}_{1}$ contains both a proton-transfer step (segment a-b) as well as OH-bond reorientation steps (b-c and c-d segments). $\mathrm{P}_{2}$, on the other hand, is purely a proton-transfer path (segment a-c).

The second distinct category includes migration paths which involve proton-transfer steps leading away from oxygen vacancies, towards more distant oxygen ions in the lattice or adjacent oxygen vacancies (paths labelled $\mathrm{P}_{3}, \mathrm{P}_{4}$ and $\mathrm{P}_{5}$ in Fig. 4). These paths are of longer

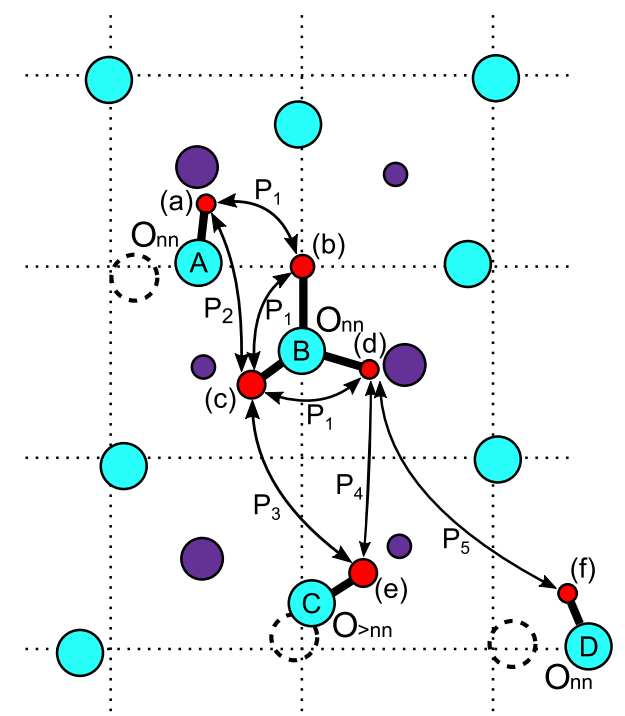

Fig. 4. Representative proton migration pathways in the bulk lattice of cubic YSZ near an oxygen vacancy (site (b)). Two-sided arrows denote the proton paths during the migration process. The lowercase symbols (a), (b), (c), (d), (e) and (f) denote minimum-energy proton sites. The migration paths $P_{1}$ and $P_{2}$ are near-vacancy paths whereas the paths $P_{3}$, $\mathrm{P}_{4}$ and $\mathrm{P}_{5}$ are vacancy-escape paths. Oxygen ion $\mathrm{O}_{\mathrm{D}}$ is denoted as $\mathrm{O}_{n n}$ because it belongs to the nn shell of an oxygen vacancy (in the lower right of the figure; not shown). 


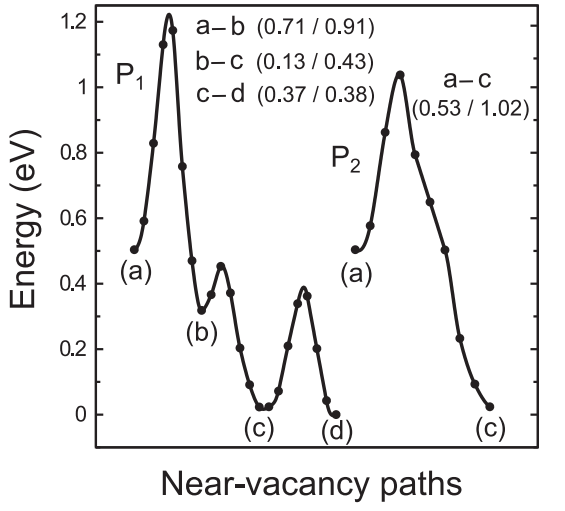

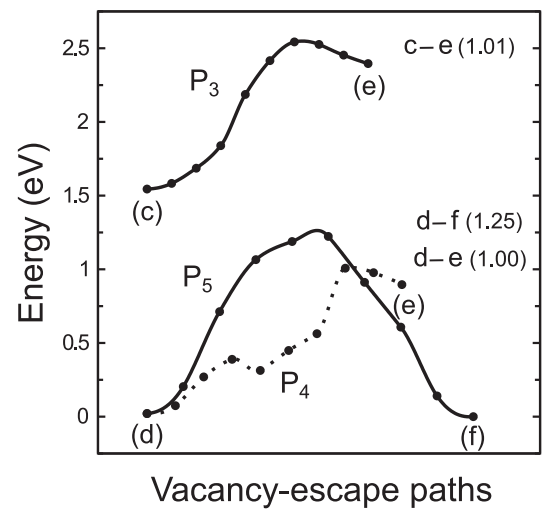

Fig. 5. Energy profiles and barriers (in $\mathrm{eV}$ ) for proton migration in the bulk lattice of YSZ. The lowercase letters in parentheses denote minimum-energy proton sites (see Fig. 4). The barriers are listed for each proton jump inside parentheses, with the forward jump listed first (proton motion from left to right) and the backward jump listed second. $P_{1}$ and $P_{2}$ are near-vacancy paths. $\mathrm{P}_{3}, \mathrm{P}_{4}$ and $\mathrm{P}_{5}$ are vacancy-escape paths. The paths are those depicted in Fig. 4. The curves were used to guide the eye and connect the exact NEB results (filled circles). range, whereby the protons escape from their nearest oxygen vacancy and hop away from them. Therefore, they contribute to a continuity of the diffusing pathways throughout the bulk lattice and are essential to macroscopic proton diffusion. They will be referred to as vacancy-escape paths from now on.

The proton migration-energy profiles were determined for both near-vacancy and vacancy-escape paths by means of the NEB method; they are shown in Fig. 5 together with the corresponding energy barriers. For the near-vacancy paths the magnitudes of the energy barriers are listed for either direction, namely for both forward jumps (right-toleft) and backward jumps (left-to-right) of the protons. The maximum barriers encountered in the different pathways are also listed in Table 1.

Starting with the near-vacancy results it can be concluded that bond-reorientation steps (b-c and $c-d$ ) possess smaller barriers with respect to the proton-transfer steps a-b and a-c. The former are in the $0.13-0.43 \mathrm{eV}$ range, whereas the latter are higher, with corresponding magnitudes ranging from 0.53 to $1.02 \mathrm{eV}$. It is also important to recognize that the specific magnitudes of the proton-transfer steps depend upon the energy of the intermediate proton sites (labelled by the lowercase letters) since these are not isoenergetic. Nonetheless, the obtained values show that migration of protons by a transfer mechanism requires large activation energies.

Even larger barriers, within a range from 1.00 to $1.25 \mathrm{eV}$ were determined for the vacancy-escape proton paths (see Fig. 5). Such a large magnitude for these barriers shows the potency of the oxygen vacancies to act as strong trapping regions for protons in YSZ, at least for the specific yttria doping studied here. The cause for such a behavior very likely originates from the peculiar distortions of the oxygen sublattice in YSZ and, in particular, the magnitude of the oxygen-oxygen O-O distances which are involved in the proton jumps. These O-O distances are rather large especially for the vacancy-escape paths and their magnitude appears to be intimately connected with the large barriers encountered for these paths.

The O-O distances in YSZ are listed in Table 1 together with the largest migration-energy barriers obtained for the corresponding migration pathways. For the vacancy-escape paths $\left(\mathrm{P}_{3}, \mathrm{P}_{4}\right.$ and $\left.\mathrm{P}_{5}\right)$ the $\mathrm{O}-\mathrm{O}$ distances are clearly larger to the O-O distances of the ideal fluorite

\section{Table 1}

Largest migration-energy barriers $\mathrm{E}_{\text {barrier }}$ (in eV) and O-O distances (in $\AA$ ) of the oxygen pairs involved in the proton paths depicted in Fig. 4 for bulk YSZ. The corresponding distances are listed for the cases where there is a proton present as well for the case when no proton exists (in parentheses). In the former case, $\mathrm{H}^{+}$is at site $c$ and bonds with $\mathrm{O}_{\mathrm{B}}$ (seeFigs. 1 and 4). $n n$ and $2 n n$ denote the O-O associations in the ideal fluorite cubic phase with corresponding O-O distances equal to $2.59 \AA$ and $3.66 \AA$, respectively.

\begin{tabular}{lccc}
\hline Paths & Oxygen pair & O-O distances with $\mathrm{H}^{+}\left({\left.\text {no } \mathrm{H}^{+}\right)}\right.$ & $\mathrm{E}_{\text {barrier }}$ \\
\hline $\mathrm{P}_{1}, \mathrm{P}_{2}$ & $\mathrm{O}_{B}-\mathrm{O}_{A} 2 \mathrm{nn}$ & $2.64(3.16)$ & 1.02 \\
$\mathrm{P}_{3}, \mathrm{P}_{4}$ & $\mathrm{O}_{B}-\mathrm{O}_{C} \mathrm{nn}$ & $3.80(2.86)$ & 1.01 \\
$\mathrm{P}_{5}$ & $\mathrm{O}_{B}-\mathrm{O}_{D} 2 \mathrm{nn}$ & $5.02(4.00)$ & 1.25 \\
\hline
\end{tabular}

cubic phase. The latter are equal to $2.59 \AA$ and $3.66 \AA$ between $n n$ and $2 n n$ oxygen neighbors, respectively. The presence of the proton further increases $\mathrm{O}-\mathrm{O}$ separations for the oxygen pairs participating in the proton-hopping events (see Table 1). This should hardly be a surprise since proton-transfer reactions in oxides are known to be coupled to the local dynamics of the oxygen sublattice [67]. For instance, MD calculations in proton-conducting perovskites showed that the energy barriers for proton transfer are intimately modulated by the O-O distances [68]. Very similar conclusions were reached in empirical calculations of proton-transfer jumps in acceptor-doped $\mathrm{CaZrO}_{3}[69]$ and $\mathrm{BaZrO}_{3}[70]$.

Table 1 lists the corresponding O-O distances for the specific O-O pairs involved in all P-type proton paths shown in Fig. 4. For the case when there is a proton in the supercell, the specific configuration considered is the one where the proton resides at site $(c)$ in Fig. 4 binding to the central oxygen ion $\mathrm{O}_{\mathrm{B}}$. It is clear that the presence of the proton increases considerably the interionic O-O distances for the vacancy-escape paths $\mathrm{P}_{3}, \mathrm{P}_{4}$ and $\mathrm{P}_{5}$. These distances are the separations between the reference ion $\mathrm{O}_{B}$ and each of its neighbors $\mathrm{O}_{C}$ and $\mathrm{O}_{D}$ further away from the central vacancy. As a measure of comparison, the O-O distances quoted in Table 1 for the $n n$ vacancy-escape paths even without a proton present $(2.86 \AA)$ are larger than the O-O distances in proton-transfer jumps in well-known proton conductors: in Yttriumdoped $\mathrm{BaZrO}_{3}$ the lengths of O-O edges participating in intraoctahedral (either $\mathrm{ZrO}_{6}$ or $\mathrm{YO}_{6}$ octahedra) and interoctahedral proton transfers lie in the 2.50-2.60 $\AA$ range [70]. For $\mathrm{CaZrO}_{3}$, the O-O distances for oxygen pairs participating in proton jumps are in the $2.75-2.91 \AA$ range and even decrease to less than $2.5 \AA$ in order to facilitate proton transfer [69].

The obtained barriers for proton migration in bulk YSZ determined here are consistently larger to reported activation energies for proton diffusion in known proton-conducting solids. Calculated proton-migration barriers of proton transfer in acceptor-doped $\mathrm{BaZrO}_{3}$ by both empirical and first-principles methodologies were less than $0.50 \mathrm{eV}$ [70,71]. Molecular dynamics calculations [72] for acceptor-doped $\mathrm{SrTiO}_{3}$ and $\mathrm{CaTiO}_{3}$ determined activation energies for proton diffusion in the $0.40-0.50 \mathrm{eV}$ range. For acceptor-doped $\mathrm{BaCeO}_{3}$ and $\mathrm{BaZrO}_{3}$ and titanates, activation energies in the $0.42-0.83 \mathrm{eV}$ range were reported by both theory and experiments [68]. Empirical calculations [69] of proton migration in $\mathrm{CaZrO}_{3}$ led to a maximum barrier of $0.74 \mathrm{eV}$.

Given the fact that vacancy-escape paths are essential for the continuity of proton pathways in the lattice and long-range macroscopic diffusion, it should be expected that they are the rate-limiting steps for proton diffusion in bulk YSZ with energy barriers within a range from 1.00 to $1.25 \mathrm{eV}$ (see Table 1 ).

\subsection{Proton sites and migration pathways in the $55(310)$ grain boundary}

The equilibrium structure of the $\Sigma 5(310)$ tilt GB obtained after energy minimization is shown in Fig. 6 with a view parallel to the tiltrotation [001] axis. The in-plane dimensions are equal to $2 \mathrm{a}_{\text {latt }}$ and 


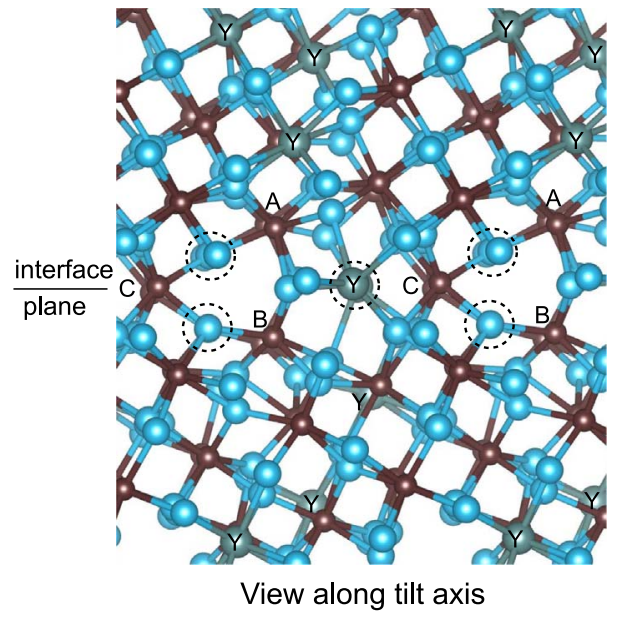

Fig. 6. Atomistic structure of the $\Sigma 5(310) \mathrm{GB}$ after structural relaxation. The interface plane is defined by the position of the $\mathrm{Zr} \mathrm{C}$ column and yttrium column (Y). The dashed circles denote the location of the Schottky defects (anion and cation vacancies). The view is along the tilt [001] axis.

$(\sqrt{10} / 2) \mathrm{a}_{\text {latt }}$ along [001] and [310], respectively. Similarly to the bulk YSZ lattice the distortion that the oxygen sublattice sustains due to doping is larger. The cation sublattice, instead, is perturbed to a much lesser degree even up to the interface plane. The geometric location of this plane is defined by the position of two cation columns in the middle: one $\mathrm{Zr}$ column denoted by $\mathrm{C}$ and one $\mathrm{Y}$ column depicted by the dashed circle (see Fig. 6). It can also be seen that the cation sublattice remains nearly symmetric with respect to the interface plane. The atomistic structure of the relaxed GB shown in Fig. 6 also agrees with the experimental observations $[33,36,38]$.

Nonetheless, the very existence of the GB creates a unique stacking sequence for the cation planes directly at the GB core and as a result some of the $\mathrm{Zr}-\mathrm{Zr}$ distances deviate considerably from the corresponding distances in the bulk crystal. In particular, in the cation A-B-C triangle at the core (see Fig. 6) the A-B edge is appreciably compressed; the $\mathrm{Zr}_{\mathrm{A}}-\mathrm{Zr}_{\mathrm{B}}$ length is equal to $3.18 \AA$, much smaller to $3.66 \AA$, the corresponding $\mathrm{Zr}-\mathrm{Zr}$ smallest distance in the bulk cubic YSZ lattice. In contrast, the two other edges are much closer to the bulk $\mathrm{Zr}-\mathrm{Zr}$ distance: the $\mathrm{Zr}_{\mathrm{A}}-\mathrm{Zr}_{\mathrm{C}}$ and $\mathrm{Zr}_{\mathrm{B}}-\mathrm{Zr}_{\mathrm{C}}$ lengths are equal to $3.67 \AA$ and $3.82 \AA$, respectively. Such short $\mathrm{Zr}-\mathrm{Zr}$ distances at the GB core are very likely the reason for the high energy of this high-angle GB [37]. Detailed calculations for the same GB in zirconia [34-37] and GBs in other oxides such as alumina [73] showed that unfavorable cation stacking at the core of interfaces in ionic materials cannot be avoided by rigid-body translations of the adjoining grains. Instead, it is a structural feature which characterizes the atomistic structure of the core of high-angle GBs in ionic materials in general [74].

In accordance with previous empirical and first-principles studies [34,36-38] it was found necessary to introduce Schottky-type defects at the GB core (cation and anion vacancies) in order to attain a stable minimum-energy configuration. All the ion columns denoted by dashed circles in Fig. 6 are half filled. These defects are part of the equilibrium GB structure and should not be viewed as chemical defects which normally increase the GB excess energies. Instead, their presence is intimately connected with the lowest-energy configurations of these high-angle boundaries. A direct consequence of their presence at the GB core is that the ionic density becomes smaller from that in the bulk crystal, with several ions of both $\mathrm{O}$ and $\mathrm{Zr}$ sublattices undercoordinated.

The type of hydrogen configurations that can exist at the core of GBs in zirconia has been studied in the past by both empirical [75] and firstprinciples [38] methods. In particular, the calculations with the empirical potentials were performed for the $55(310) \mathrm{GB}$ in pure cubic zirconia and showed that hydration energies were much lower in the GB region. Thus, a higher concentration of protons with respect to the

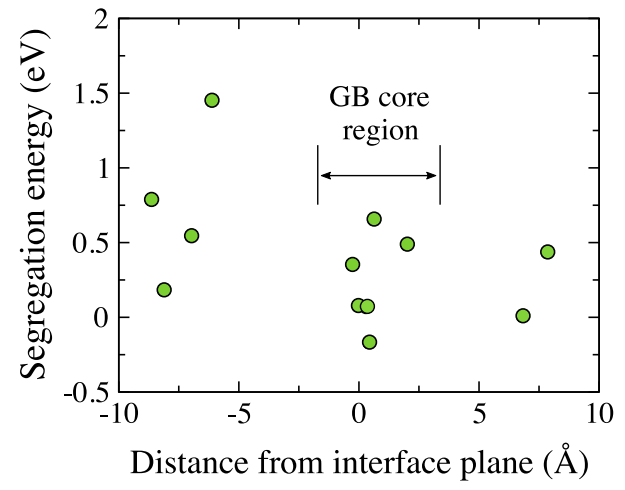

Fig. 7. Total energies of proton configurations as a function of distance from the interface plane of the $\Sigma 5(310) \mathrm{GB}$. The reference energy is the one of the lowest-energy proton configuration in the bulk region of the supercell.

bulk would be expected [75].

The strategy in the present work was to introduce the protons at various positions in the GB supercell, sampling both bulk-like regions and regions near the GB core. Similarly to the bulk YSZ, inspection of the final magnitude of the total energies of the proton configurations displayed a range of energies. For twelve of these configurations (six within the GB-core region and six at the bulk-like region) the corresponding energies are plotted in Fig. 7. From the calculated energies, the segregation energy for a particular proton configuration bound in the GB-core region could then be obtained as the difference between the formation energy of this configuration and the formation energy for a proton residing in the bulk-like region of the supercell:

$E^{\text {segr }}\left(\mathrm{H}^{+}\right)=E_{\mathrm{GB}}^{f}\left(\mathrm{H}^{+}\right)-E_{\text {bulk }}^{f}\left(\mathrm{H}^{+}\right)$

The problem, however, for such a definition is that proton energies in bulk YSZ are not isoenergetic owing to the disorderded structure of the bulk-crystalline YSZ lattice; instead, they exhibit appreciable variations in magnitude depending on the specific site (see Fig. 7). For the segregation energies presented in Fig. 7 the energy reference for a bulk energy was the one of the lowest-energy proton configuration encountered in the bulk region.

Alternatively, by taking an average of the proton energies determined in the bulk regions it can be concluded that segregation energies in the GB are about $-0.5 \mathrm{eV}$ per atom, thus indicating a marginal energy gain for protons to segregate to the GB core. This finding is in disagreement with recent DFT calculations for proton impurities in a high-angle $\mathrm{GB}$ in $\mathrm{MgO}$ where protons displayed strong segregation propensity; segregation energies as large as $-1.78 \mathrm{eV}$ were reported [76]. This contrasting behavior very likely originates from the ability of the bulk YSZ lattice to absorb the resulting perturbations following proton incorporation. Indeed, YSZ is an extremely flexible oxide host which can sustain much larger distortions in the proton neighborhood (with ionic displacements as large as $1 \AA$ ) with respect to crystalline $\mathrm{MgO}$ or monoclinic zirconia [19].

In order to assess the GB capacity to aid (or hinder) proton transport, migration-energy profiles were determined for selected proton pathways at the GB-core region. First, a proton-migration pathway was chosen which traverses laterally the GB interfacial region and remains confined in the GB core. This pathway comprises three distinct protontransfer steps (proton jumps denoted by the segments 1-2, 2-3 and 3-4 in Fig. 8) which are characterized by dominant in-plane displacements of the migrating proton. Paths 1-2 and 3-4 are long and amount to total proton displacements of $3.35 \AA$ and $3.91 \AA$, respectively. In contrast, the 2-3 segment is considerably shorter with a proton displacement equal to $0.88 \AA$. It is also important to note that the path 3-4 connects proton positions separated by the A-B-C cation triangle, therefore, it requires protons to pass through it in order to complete the diffusive jump. The point of view adopted in the present study is that such cation 


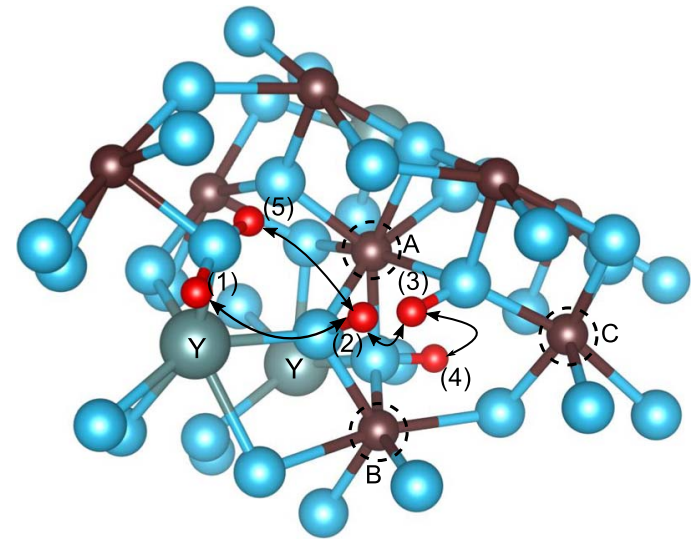

Fig. 8. Proton configurations and migration pathways at the core region of the $\Sigma 5(310)$ GB. Numbered sites denote the positions of the protons (in red) obtained after energy minimization. Migration paths (proton jumps) are shown as two-sided arrows. Dashed circles denote the $\mathrm{Zr}$ ions making up the cation triangle (A-B-C) (see Fig. 6). (For interpretation of the references to color in this figure legend, the reader is referred to the web version of this article.)

clusters (cation triangles for the specific GB here) can become strong obstacles to proton transport; first, their mere existence creates regions within the GB cores virtually inaccessible to protons, owing to strong repulsive interactions of electrostatic origin. Secondly, they can increase the necessary activation energy that protons have to overcome in order to cross these regions.

Furthermore, another proton path was selected (denoted by the $2-5$ segment in Fig. 8) which connects proton positions inside the GB-core region and the bulk interior. The associated migration energy would indicate the ease of access of the GB region for a proton migrating from the bulk (and vice versa). The total proton displacement for this path amounts to $2.50 \AA$ and possesses a strong out-of-plane character.

The corresponding migration-energy profiles are shown in Fig. 9. It

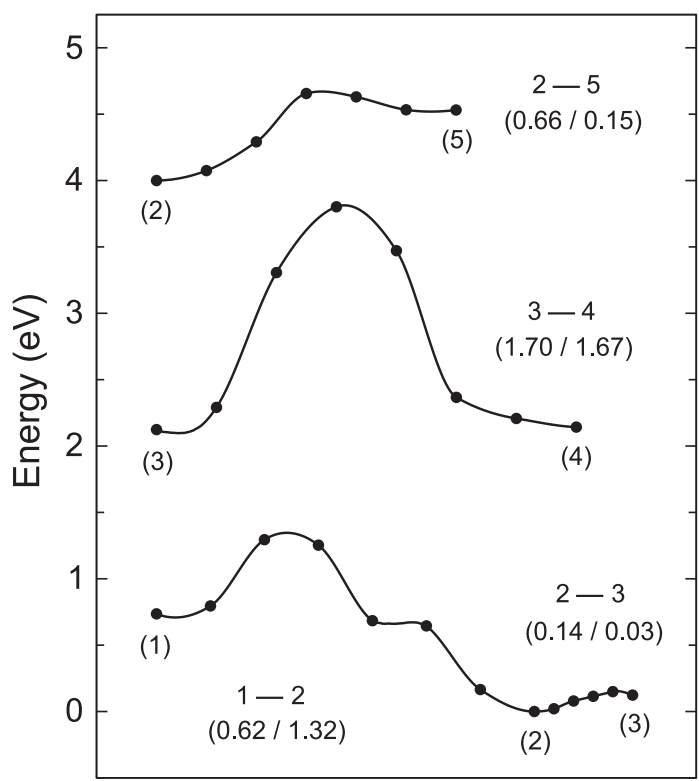

\section{Grain boundary paths}

Fig. 9. Energy profiles and barriers (in $\mathrm{eV}$ ) for proton migration at the core of the $\Sigma 5(310)$ GB. Numbered sites in parentheses denote minimum-energy proton sites in the GB core (see Fig. 8). The specific paths $1-2,2-3,3-4$ and $2-5$ involve individual proton jumps and are depicted in Fig. 8. The barriers are listed for each proton jump inside parentheses, with the forward jump listed first (proton motion from left to right) and the backward jump listed second. The curves were used to guide the eye and connect the exact NEB results (filled circles). can be seen that very small barriers are obtained for the shortest 2-3 path $(0.14 \mathrm{eV}$ for the forward jump and $0.03 \mathrm{eV}$ for the backward). This shows that the disordered GB-core structure can lead to easy (albeit localized) proton hopping with considerably smaller barriers than those experienced by protons migrating in the bulk crystal. The barrier for the forward 1-2 jump is also rather moderate $(0.62 \mathrm{eV})$; in contrast, the barrier for the same path but of the reverse sense (namely 2-1) is much larger (equal to $1.32 \mathrm{eV}$ ), something which originates from the fact that the site (1) of the proton has a higher energy. In stark contrast, the path 3-4 where the proton traverses the cation A-B-C triangle is characterized by very large barriers irrespective of the proton coming from the left or the right. Since both sites (3) and (4) are nearly iso-energetic such high magnitudes for the barriers probably originate from the strong repulsion that the proton should experience while attempting to pass through the triangle of the positively charged $\mathrm{Zr}$ ions (each of ionic charge +4 ). This result shows that unfavorable cation arrangements of this type within GB cores can be strong obstacles to proton motion. For the out-of-plane 2-5 path there is a striking asymmetry in the energy barriers; they were found equal to $0.66 \mathrm{eV}$ and $0.15 \mathrm{eV}$ for the forward and backward jumps, respectively.

The present results bear certain similarities to calculations by Kim et al. [77] who determined energy barriers for proton migration in Yttrium-doped $\mathrm{BaZrO}_{3}$. For the case of a high-angle GB in this perovskite they identified proton pathways at the GB core with very high energy barriers. Their assessment was that the existence of such pathways in the GB regions would severely restrict proton conduction in polycrystalline Yttrium-doped $\mathrm{BaZrO}_{3}$. Likewise, molecular-dynamics simulations of protons at a GB in Yttrium-doped $\mathrm{BaZrO}_{3}$ showed severely reduced proton mobility in the GB core [78] suggesting that the GB acts as an obstacle to proton motion. These calculations are in line with experimental observations for the same material where a high GB resistance to proton transport was reported [79].

Furthermore, it is also relevant to mention that GB cores in acceptor-doped zirconia are positively charged, thus favoring segregation of negatively-charged trivalent solute atoms [46]. In this case they would act as stronger obstacles to proton motion approaching from the bulk regions of YSZ; therefore even larger energy barriers would be required for protons to migrate across the GBs.

\section{Conclusions}

The aim of the present study was two-fold: to determine the energetics and sites of protons in cubic YSZ from the relevant defectformation energies and secondly to calculate energy barriers for proton migration. The role of oxygen vacancies and GBs, two major defects in YSZ, was specifically examined. The calculated hydration energy at $0 \mathrm{~K}$ was found to be negative but small, something which indicates a minimal uptake of protonic defects from the surrounding water vapor. Estimates of the equilibrium proton concentration were also obtained for finite temperatures.

Oxygen vacancies were seen to behave as localized trapping regions whereby protons bind more favorably, forming bond-type $\mathrm{O}_{\mathrm{nn}} \mathrm{H}$ configurations of lower formation energy (with $\mathrm{O}_{\mathrm{nn}}$ being the nearestneighbor oxygen ions to the vacancies). Invariably, the $\mathrm{O}_{\mathrm{nn}}-\mathrm{H}$ bond formation entailed strong structural relaxations with the breaking of $\mathrm{Zr}$ $\mathrm{O}$ bonds and eventually leading to a smaller (two-fold) $\mathrm{Zr}$ coordination for the $\mathrm{O}_{\mathrm{nn}}$ 's. This specific peculiarity in the relaxation pattern increases the interionic O-O distances between the $\mathrm{O}_{\mathrm{nn}}$ ions and their oxygen neighbors further away from the vacancy, effectively also increasing the energy barriers for the corresponding proton-transfer steps.

A distinction of proton pathways in the lattice was made according to their proximity to oxygen vacancies. These are (a) near-vacancy paths with proton hopping occurring exclusively between oxygen ions which are nearest-neighbors to the vacancies, and (b) vacancy-escape paths which contribute to long-range macroscopic proton conduction. The OH-bond reorientation step with activation energies less than 
$0.50 \mathrm{eV}$ was found to be the energetically preferable mode for proton migration near oxygen-vacancy sites. Instead, proton-transfer steps required activation energies in excess of $0.50 \mathrm{eV}$ for near-vacancy hops. Even higher barriers were obtained for the vacancy-escape pathways (larger than $1 \mathrm{eV}$ ), a consequence of the larger O-O distances involved. These results suggest that vacancy-escape proton-transfer paths are the rate-limiting steps for proton diffusion in bulk-crystalline YSZ, considering also the fact that protons need to cross oxygen vacancies in order to achieve pathway continuity for long-range diffusion throughout the oxide. Taking everything into account, the present results cast serious doubts on the ability of the bulk-crystalline YSZ lattice to yield high proton conductivity at low and intermediate temperatures.

Concerning the $\Sigma 5(310) \mathrm{GB}$, the present calculations showed a marginal energy gain for proton segregation to the GB core. Migration calculations for distinct proton pathways within the GB-core region led to strong variations in the magnitude of the energy barriers, something that could partially be explained from the different energies of the proton sites within the core. Additionally, the presence of unfavorable cation stacking in the GB core with closely-spaced $\mathrm{Zr}$-Zr pairs had an impact on proton migration: very high activation energies were required by protons to cross $\mathrm{Zr}$-triangle arrangements in the core region. This will clearly have an effect on proton mobility increasing resistance to proton transport.

\section{Acknowledgments}

This work was supported with funds from (i) FEDER (Programa Operacional Factores de Competitividade COMPETE) and from FCT Portugal - Fundação para a Ciência e Tecnologia under the UID/FIS/ 04564/2016 and PTDC/FIS/102722/2008 projects. The computer resources of the Department of Physics of the University of Coimbra were used, including the Milipeia cluster at the Laboratory for Advanced Computing. The author thanks Rui Vilão, Ricardo Vieira, Stephen Cox, Alois Weidinger, Helena Alberto, João Gil and Paulo Gordo for fruitful discussions.

\section{Appendix A}

The construction of the various YSZ supercells consisted of introducing yttrium atoms substitutionally in the cation sublattice. The chargecompensating oxygen vacancies were then created for various initial vacancy-vacancy distances. The total energies of all distinct YSZ supercells are presented in Fig. 10 as a function of the closest-spaced vacancy-vacancy pair in the respective cell. The energy difference between the highest- and lowest-energy supercells amounted to about $1.5 \mathrm{eV}$. It was observed that oxygen vacancies mutually repelled each other thus avoiding to form firstnearest neighbor $(1 \mathrm{nn})$ pairs in the anion sublattice. Only one configuration of such type persisted during structural relaxation and it possessed high energy (see Fig. 10). Similarly, in all minimum-energy supercells no fourth-nearest neighbor (4nn) vacancy-vacancy pairs existed.

In the lowest-energy supercell (which defines the energy zero in Fig. 10) the closest-spaced oxygen vacancies were fifth-nearest neighbors (5nn). The yttrium substitutional defects displayed a preference to occupy next-nearest neighbor positions with respect to the vacancies, in agreement with earlier results for YSZ with the same yttria content [27-32]. Nonetheless, nearest-neighbor yttrium-vacancy pairs were also found to exist in some of the stable supercells. The multiplicity of such pairs is explicitly shown for each supercell in Fig. 10.

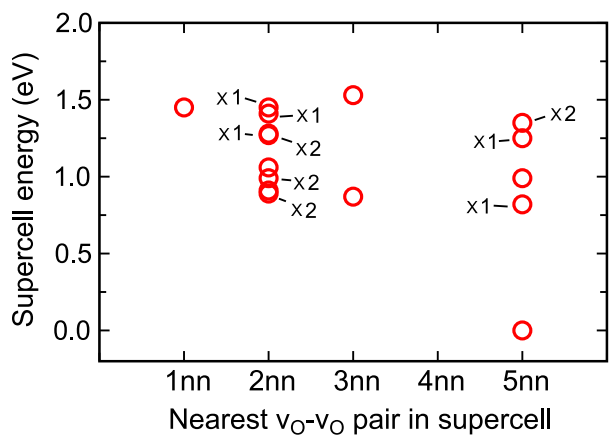

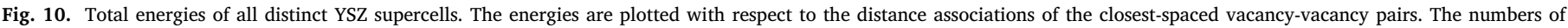
nearest-neighbor yttrium-vacancy pairs for each configuration are explicitly shown. The energy zero is taken as the total energy of the lowest-energy supercell.

\section{References}

[1] A. Heuer, L.W. Hobbs, Science and Technology of Zirconia, Advances in Ceramics, vol. 3, The American Ceramic Society, Westerville, OH, 1981.

[2] J.R. Brandon, R. Taylor, Surf. Coat. Tech. 46 (1991) 75-90.

[3] R. Ramamoorthy, P. Dutta, S.A. Akbar, J. Mater. Sci. 38 (2003) 4271-4282.

[4] S.P.S. Badwal, Solid State Ion. 52 (1992) 23-32.

[5] N.Q. Minh, J. Am. Ceram. Soc. 76 (1993) 563-588.

[6] M.O. Zacate, L. Minervini, D.J. Bradfield, R.W. Grimes, K.E. Sickafus, Solid State Ion. 128 (2000) 243-254.

[7] S. Kim, U. Anselmi-Tamburini, H.J. Park, M. Martin, Z.A. Munir, Adv. Mater. 20 (2008) 556-559.

[8] S. Kim, H.J. Avila-Paredes, S. Wang, C.T. Chen, R.A. De Souza, M. Martin, Z.A. Munir, Phys. Chem. Chem. Phys. 11 (2009) 3035-3038.

[9] G. Chiodelli, F. Maglia, U. Anselmi-Tamburini, Z.A. Munir, Solid State Ion. 180 (2009) 297-301.

[10] J.S. Park, Y.B. Kim, J.H. Shim, S. Kang, T.M. Gür, F.B. Prinz, Chem. Mater. 22 (2010) 5366-5370.

[11] H.J. Avila-Paredes, J. Zhao, S. Wang, M. Pietrowski, R.A. De Souza, A. Reinholdt, Z.A. Munir, M. Martin, S. Kim, J. Mater. Chem. 20 (2010) 990-994.

[12] B. Scherrer, M.V.F. Schlupp, D. Stender, J. Martynczuk, J.G. Grolig, H. Ma, P.
Kocher, T. Lippert, M. Prestat, L.J. Gauckler, Adv. Funct. Mater. 23 (2013) 1957-1964.

[13] S. Raz, K. Sasaki, J. Maier, I. Riess, Solid State Ion. 143 (2001) 181-204.

[14] C. Tandé, D. Pérez-Coll, G.C. Mather, J. Mater. Chem. 22 (2012) 11208-11213.

[15] S. Stotz, C. Wagner, Ber. Bunsen. Phys. Chem. 70 (1966) 781-788.

[16] C. Wagner, Ber. Bunsen. Phys. Chem. 72 (1968) 778-781.

[17] S.F.J. Cox, J.L. Gavartin, J.S. Lord, S.P. Cottrell, J.M. Gil, H.V. Alberto, J. Piroto Duarte, R.C. Vilão, N. Ayres de Campos, D.J. Keeble, E.A. Davis, M. Charlton, D.P. van der Werf, J. Phys.: Condens. Matter 18 (2006) 1079-1119.

[18] R.B.L. Vieira, R.C. Vilão, A.G. Marinopoulos, P.M. Gordo, J.A. Paixão, H.V. Alberto, J.M. Gil, A. Weidinger, R.L. Lichti, B. Baker, P.W. Mengyan, J.S. Lord, Phys. Rev. B 94 (2016) 115207.

[19] A.G. Marinopoulos, Phys. Rev. B 86 (2012) 155144.

[20] P. Hohenberg, W. Kohn, Phys. Rev. 136 (1964) B864-B871.

[21] W. Kohn, L.J. Sham, Phys. Rev. 140 (1965) A1133-A1138.

[22] K.D. Kreuer, A. Fuchs, J. Maier, Solid State Ion. 77 (1995) 157-162.

[23] K.D. Kreuer, Annu. Rev. Mater. Res. 33 (2003) 333-359.

[24] Y.A. Mantz, R.S. Gemmen, J. Phys. Chem. C 114 (2010) 8014-8025.

[25] J. Rabone, P. Van Uffelen, J. Nucl. Mater. 459 (2015) 30-36.

[26] J.A. Dawson, H. Chen, I. Tanaka, Phys. Chem. Chem. Phys. 16 (2014) 4814-4822.

[27] G. Stapper, M. Bernasconi, N. Nicoloso, M. Parrinello, Phys. Rev. B 59 (1999) 797-810. 
[28] A. Bogicevic, C. Wolverton, G.M. Crosbie, E.B. Stechel, Phys. Rev. B 64 (2001) 014106.

[29] S. Ostanin, A.J. Craven, D.W. McComb, D. Vlachos, A. Alavi, A.T. Paxton, M.W. Finnis, Phys. Rev. B 65 (2002) 224109.

[30] R. Pornprasertsuk, P. Ramanarayanan, C.B. Musgrave, F.B. Prinz, J. Appl. Phys 98 (2005) 103513.

[31] F. Pietrucci, M. Bernasconi, C. Di Valentin, F. Mauri, C.J. Pickard, Phys. Rev. B 73 (2006) 134112.

[32] F. Pietrucci, M. Bernasconi, A. Laio, M. Parrinello, Phys. Rev. B 78 (2008) 094301.

[33] E.C. Dickey, X. Fan, S.J. Pennycook, J. Am. Ceram. Soc. 84 (2001) 1361-1368.

[34] C.A.J. Fisher, H. Matsubara, Solid State Ion. 113-115 (1998) 311-318.

[35] T. Oyama, M. Yoshiya, H. Matsubara, K. Matsunaga, Phys. Rev. B 71 (2005) 224105.

[36] Z. Mao, S.B. Sinnott, E.C. Dickey, J. Am. Ceram. Soc. 85 (2002) 1594-1600.

[37] A.G. Marinopoulos, J. Phys: Condens. Matter 23 (2011) 085005.

[38] A.G. Marinopoulos, J. Phys.: Condens. Matter 26 (2014) 025502.

[39] P.E. Blöchl, Phys. Rev. B 50 (1994) 17953-17979.

[40] G. Kresse, D. Joubert, Phys. Rev. B 59 (1999) 1758-1775.

[41] J.P. Perdew, K. Burke, M. Ernzerhof, Phys. Rev. Lett. 77 (1996) 3865-3868.

[42] G. Kresse, J. Hafner, Phys. Rev. B 47 (1993) 558-561.

[43] G. Kresse, J. Hafner, Phys. Rev. B 49 (1994) 14251-14269.

[44] G. Kresse, J. Furthmüller, Phys. Rev. B 54 (1996) 11169-11186.

[45] H. Monkhorst, J. Pack, Phys. Rev. B 13 (1976) 5188-5192.

[46] X. Guo, R. Waser, Prog. Mater. Sci. 51 (2006) 151-210.

[47] H. Jónsson, G. Mills, K.W. Jacobsen, B.J. Berne, G. Ciccotti, D.F. Coker (Eds.), Nudged Elastic Band Method for Finding Minimum Energy Paths of Transitions in Classical and Quantum Dynamics in Condensed Phase Simulations, World Scientific, 1998, pp. 385-404.

[48] D. Steele, B.E.F. Fender, J. Phys. C: Solid State Phys. 7 (1974) 1-11.

[49] D.N. Argyriou, M.M. Elcombe, A.C. Larson, J. Phys. Chem. Solids 57 (1996) 183-193.

[50] P.W. Peacock, J. Robertson, Appl. Phys. Lett. 83 (2003) 2025-2027.

[51] K. Xiong, J. Robertson, S.J. Clark, J. Appl. Phys 102 (2007) 083710.

[52] J.L. Lyons, A. Janotti, C.G. Van de Walle, Microelectron. Eng. 88 (2011) 1452-1456.

[53] M. Youssef, B. Yildiz, Phys. Chem. Chem. Phys. 16 (2014) 1354-1365.
[54] N.A. Richter, S. Sicolo, S.V. Levchenko, J. Sauer, M. Scheffler, Phys. Rev. Lett 111 (2013) 045502

[55] M.W. Chase Jr, NIST-JANAF Thermochemical Tables 4th Ed. American Chemical Society and American Institute of Physics for the National Institute of Standards and Technology, Woodbury NY, 1998.

[56] P.E. Blöchl, E. Smargiassi, R. Car, D.B. Laks, W. Andreoni, S.T. Pantelides, Phys. Rev. Lett. 70 (1993) 2435-2438.

[57] S. Kasamatsu, T. Tada, S. Watanabe, Solid State Ion. 183 (2011) 20-25.

[58] M. Youssef, B. Yildiz, Phys. Rev. B 86 (2012) 144109.

[59] T.S. Bjorheim, A. Kuwabara, T. Norby, J. Phys. Chem. C 117 (2013) 5919-5930.

[60] H.D. Wiemhöfer, U. Vohrer, Ber. Bunsenges. Phys. Chem. 96 (1992) 1646-1652.

[61] T. Norby, Y. Larring, Curr. Opin. Solid State Mater. Sci. 2 (1997) 593-599.

[62] T. Norby, M. Wideroe, R. Glöckner, Y. Larring, Dalton Trans. (2004) 3012-3018.

[63] H. Takahashi, I. Yashima, K. Amezawa, K. Eguchi, H. Matsumoto, H. Takamura, S. Yamaguchi, Chem. Mater. 29 (2017) 1518-1526.

[64] A. Eichler, Phys. Rev. B 64 (2001) 174103.

[65] M.E. Björketun, P.G. Sundell, G. Wahnström, Faraday Discuss. 134 (2007) 247-265.

[66] K.D. Kreuer, Chem. Mater. 8 (1996) 610-641.

[67] K.D. Kreuer, Solid State Ion. 125 (1999) 285-302.

[68] W. Munch, K.D. Kreuer, G. Seifert, J. Maier, Solid State Ion. 136-137 (2000) 183-189.

[69] M.S. Islam, R.A. Davies, J.D. Gale, Chem. Mater. 13 (2001) 2049-2055.

[70] B. Merinov, W. Goddard III, J. Chem. Phys 130 (2009) 194707.

[71] M.E. Björketun, P.G. Sundell, G. Wahnström, Phys. Rev. B 76 (2007) 054307.

[72] W. Munch, K.D. Kreuer, G. Seifert, J. Maier, Solid State Ion. 125 (1999) 39-45.

[73] A.G. Marinopoulos, S. Nufer, C. Elsässer, Phys. Rev. B 63 (2001) 165112.

[74] A.P. Sutton, R.W. Balluffi, Interfaces in Crystalline Materials, Clarendon, Oxford, 1995.

[75] J.A. Dawson, I. Tanaka, Langmuir 30 (2014) 10456-10464.

[76] K.P. McKenna, A.L. Shluger, Phys. Rev. B 79 (2009) 224116.

[77] D.-H. Kim, B.-K. Kim, Y.-C. Kim, Solid State Ion. 213 (2012) 18-21.

[78] A.C.T. van Duin, B.V. Merinov, S.S. Han, C.O. Dorso, W.A. Goddard III, J. Phys. Chem. A 112 (2008) 11414-11422.

[79] Y. Yamazaki, R. Hernandez-Sanchez, S.M. Haile, Chem. Mater. 21 (2009) 2755-2762. 